\title{
Two-wheeler Detection Algorithm Research using Homogeneity Operator and Gamma Correction based on Adaboost Classifier
}

\author{
Yeunghak Lee ${ }^{1}$, Yunju Jung ${ }^{2}$ and Jaechang Shim ${ }^{3, *}$ \\ ${ }^{1,2,3}$ Dept. of Computer Science, Andong National University \\ 1annaturu@ikw.ac.kr, ${ }^{2}$ vrjung@hanmail.net, ${ }^{3}$ jcshim@andong.ac.kr
}

\begin{abstract}
These days technology development for intelligent automobiles is accelerating more and more. This paper proposes an innovative algorithm for detecting the two wheelers with various shapes according to the viewpoints for a vision based intelligent vehicle. The images we are using have two properties, homogeneity and heterogeneity. In this paper, we use homogeneity operator which gives good rendition of edges as well as a modified histogram of oriented gradients (HOG) method which uses opposite property to extract features from the input image. HOG descriptor, which is one of the well-known methods for object detection, is a feature descriptor using edge intensity in local region. And this paper also used the periodicity of the size of spatial regions; cells and blocks, to improve the detection rate. Contrary to concatenating feature vectors using a dense grid of HOG, proposed algorithm storage extracted feature vectors according to the period in each step. The Adaboost classification algorithm makes strong classification from weak classification of extracted feature vectors (positive and negative vectors). This proposed system maintains the simplicity of evaluation of traditional formulation while being more discriminative. Our experimental results show that detection of a two-wheeler based on the proposed algorithm leads to higher detection accuracy than the conventional methods.
\end{abstract}

Keywords: Homogeneity, Histogram of oriented gradients, Adaboost, Intelligent vehicle, Homogeneity operator

\section{Introduction}

Many kinds of transportation systems have been developed to help the people to move things or to pursue the convenience of human life. Changes in computer science and information technology environment are developing very rapidly because of the development of intelligent interface and multimedia. Especially in intelligent vehicles, but while a simple device or vehicle to move things may be in the past, the shape (or style) and function is very fast and this variety has progressed to the support of MEMS, FPGA, embedded system and etc., in now [1].

In the past, a vehicle was regarded as a simple tool to transport people and commodities. However, the shapes and functions of vehicles continue to develop rapidly and in diverse ways. The development of vehicles was concentrated on not only improving performance, but also to protect the drivers and passengers in a vehicle in the occurrence of a traffic accident. The degree of injury to passengers in a vehicle caused by a traffic accident has been reduced gradually using the advantage of many safety device techniques mounted in and outside of the vehicle. But the accidents outside of a vehicle that are caused by a driver's carelessness or by road environments still pose many problems, so it is required to detect the risk of a traffic accident in order to save lives [2].

Received (May 16, 2017), Review Result (August 25, 2017), Accepted (September 15, 2017) 
The development for the safety device design of vehicles, especially airbags, bumpers, electronic equipment, etc., has been focused on protecting drivers from accidents on the road and enhancing comfortability until a few years ago. Most of the research for the safety of humans has been worked to improve the detection rate for pedestrians and vehicles on the road [2-3]. Lately, the vulnerable road users (VRUs), consisting of humans, bicyclists, two wheelers, and other small vehicles, detection has been researched to protect against accident for intelligent vehicles [1].

Most of the studies relating to human safety over the past several years have mainly concentrated on increasing the detection rate of pedestrians and automobiles on a road from a still image and framework. The ability to detect the two-wheeler is a task that human safety risibly performs but one that computers to date have been unable to perform robustly. Now, the study scope has expanded to protect vulnerable road users (VRUs) such as small automobiles [2]. Because pedestrians and bicycle riders are very vulnerable road participants among VRUs, they are a hot subject for the study field in an intelligent transportation system. Therefore, various types of sensors are utilized for an accurate and real time detection and tracking; NIR, FIR, LIDAR, RADAR, Laser Scanner, fusion system, and etc.,

Among VRUs on the road, pedestrians are the slowest while the others have the characteristics of moving speedily. Two-wheelers, differ from ordinary pedestrians, and are expressed with complicated shapes in combinations of the rider's dressing style, hair style, whole body attitude, forms and patterns of loading, and various types of twowheelers, which makes the shapes even further complicated according to the viewing angle, so that it requires a stronger algorithm to detect changes in their shapes.

The automobile vision-based systems have mainly concentrated on recognizing pedestrians and automobiles [3]. As stated in the foregoing, detection of two-wheelers on the road is similar to that of pedestrians on the road. Extracting characteristics for detecting pedestrians is classified with single characteristics, multiple characteristics, whole area characteristics, and district characteristics pursuant to the usage of extracted characteristics [4]. Especially, HOG characteristics or Improved HOG characteristics are widely utilized in the methods for recognizing pedestrians by using automobile vision. Zhu et al. [5] applied the HOG characteristics based on variable block size to improve detection speed. Further, Watanabe et al. [6] utilized co-occurrence HOG characteristics, and Wang et al. [7] utilized HOG-LBP human detection to improve detection accuracy.

Generally speaking, the literature on pedestrian detection system is abundant [8]. Features can be distinguished into global features, local features, single features, and multiple features depending on how features are measured and used [9]. Principal component analysis (PCA) [10] operates global features. On the other hand, local features are extracted by dividing a sliding window into a different subset region of the image [4].

LBP has been used for texture classification and face detection as a computer efficient texture feature [11-12]. And it is very invariant for illumination and contrast to gray level changes and has good anti-noise performance. Despite the great success of LBP in early applications, its practical results are not satisfactory in several fields. Hence many researchers have improved the LBP in the specific domain and achieved lots of significant research [13].

As we mentioned previously, two wheelers similar with not only the shape of the pedestrian require a detection technique based on several features. Two wheelers consist of a human and machine; usually a human is upper part and machine is lower part in the shape. In this paper, we define that bicyclist (BL) as people riding the bicycle and motorcycle driver (MD) as people riding the motorcycle [8]. So two wheelers detection system can be adapted to the pedestrian detection algorithms for feature extraction, classification, and non-maxima suppression. The solution of slow performance from a dense encoding scheme and multi-level scale images is to use a boosting algorithm [14] to speed up classification process. Because of above reasons, we tried to use modified HOG 
and homogeneity operator algorithm to select best features and Adaboost to improve detection rate.

This paper consists of the following: Section 2 introduces basic extracting characteristic methods with modified HOG and homogeneity operator algorithm that can increase detection rates significantly. Section 3 states the framework and training of a suggested two-wheelers detecting system. The evaluation and detailed analysis for the experimental results are summarized in Section 4. Section 5 states the conclusion.

\section{Feature Extraction from Input Image}

\subsection{Histogram of Oriented Gradients (HOG)}

HOG [6] is a feature descriptor used in computer vision and image processing for the purpose of object detection. The technique counts occurrences of gradient orientation in localized portions of an image. This method is similar to that of edge orientation histograms, scale-invariant feature transform descriptors, which uses normalized local spatial histograms as a descriptor, and shape contexts, but differs in that it is computed on a dense grid of uniformly spaced cells and uses overlapping local contrast normalization for improved accuracy.

In general, it converts the distribution directions of brightness for a local region into a histogram to express them in feature vectors, which is utilized to express the shape characteristics of an object. It is influenced a little with the effect of illumination by converting the distribution of near pixels for a local region into a histogram, and has a strong feature for a geometric change of local regions. The main idea is that local object appearance and shape can often be characterized rather well by the distribution of local intensity gradients or edge directions. This is achieved by dividing the image into cells and for each cell one-dimension histogram of gradient directions over the pixels of the cell is calculated. Then each block in the image consists of a number of cells, as shown Figure 1. The method for calculating HOG feature is stated in the following.

After calculating $x, y$ derivatives ( $d x_{\text {and }} d y$ ), the magnitude $|m(x, y)|$ and orientation $\theta(x, y)$ of the gradient for each pixel $I(x, y)$ is computed from

$$
\begin{aligned}
& d x=I(x+1, y)-I(x-1, y) \\
& d y=I(x, y+1)-I(x, y-1) \\
& |m(x, y)|=\sqrt{d x^{2}+d y^{2}} \\
& \theta(x, y)=\arctan \left(\frac{d y}{d x}\right)
\end{aligned}
$$

Since unsigned orientations are desired for this implementation, the orientation range of degree which is less $0^{\circ}$ is summed up with $180^{\circ}$.

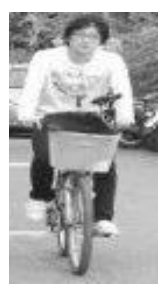

(a)

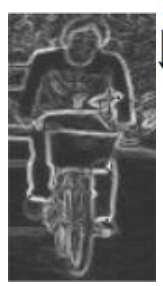

(b)

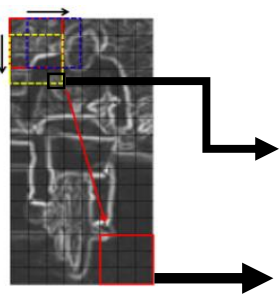

(c)

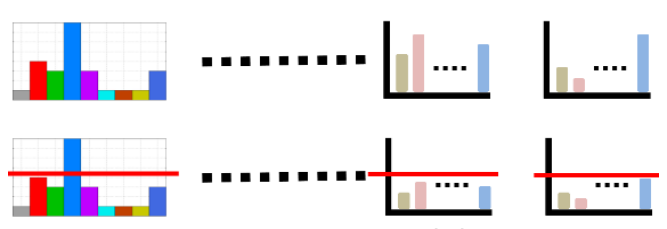

(d)

Figure 1. The Example of Two Wheelers HOG Normalization. (a) Original image (b) Calculated Magnitude Vector (c) Cells (Black Lattice) and Blocks (Red Box, 3x3 Cells), Dotted Box (Yellow and Blue) is Blocks Sliding (d) A Cell Histogram and Block Normalization 
In this paper, each cell, as shown Figure 1 (c), is represented by $8 \times 8$ pixel size and has 9 bins covering the orientation for $\left[0^{\circ}, 180^{\circ}\right]$ interval, as shown in Figure 2 (c). Directional histograms for brightness prepared in each of the cells were normalized as a block of $3 \times 3$ cells. Normalization processes are summarized in Figure 2 (c), where a movement of a block is based on that it is moved to right side and to lower side by one cell each. A contrast-normalization is used on the local responses to get better invariance regarding illumination, shading, etc., To normalize the cell's orientation histograms, it should be grouped into blocks ( $3 \times 3$ cells). This is done by accumulating a measure of local histogram value over the blocks and the result is then used to normalize the cells in the block. Although there are four different methods for block normalization suggested by Dalal and Triggs [13], L2-norm normalization $\Pi$ is implemented using equation (5)

$$
\Pi=\frac{B}{\sqrt{\|B\|_{2}^{2}+\varepsilon^{2}}}
$$

where $\varepsilon$ be small constant instead of zero for the denominator.

In this paper, the dimension number for the width and height of an input image are $128 \times 64$ pixels, the dimension number of the histogram is 9 , the size of cell is 8 , and the size of block 3, is then calculated, and HOG feature vectors with 6804 dimension is obtained.

\subsection{Homogeneity Operator}

There are many kinds of simple edge detection techniques, such as Sobel, Kirsch, Prewitt, etc., Some edge detectors may work well in one application and perform poorly in others. This paper used the simplest and quickest edge detector, called HOG to determine the maximum value from a series of pixel subtraction. The operator subtracts each of the pixels next to the center of a $3 \times 3$ window from the center pixel as shown in Figure 2 [16]. The result is the maximum of the absolute value of these subtractions, as follow.

$$
I^{\prime}(x, y)=\max \{I(x, y)-I(x+i, y+j)\}_{i, j=-1,0,+1}
$$

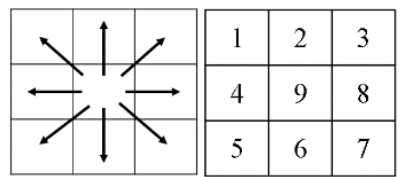

\begin{tabular}{|l|l|l|}
\hline 10 & 5 & 3 \\
\hline 10 & 5 & 3 \\
\hline 10 & 5 & 3 \\
\hline
\end{tabular}

\begin{tabular}{|c|c|c|}
\hline 20 & 20 & 20 \\
\hline 20 & 20 & 20 \\
\hline 20 & 20 & 1 \\
\hline
\end{tabular}

$\operatorname{Max}=\{|9-1|,|9-2|,|9-3|,|9-4|,|9-5|,|9-6|,|9-7|,|9-8|\}=36$

$\operatorname{Max}=\{|5-10|,|5-5|,|5-3|,|5-10|,|5-3|,|5-10|,|5-5|,|5-10|\}=5$

$\operatorname{Max}=\{|20-20|,|20-20|,|20-20|,|20-20|,|20-20|,|20-20|,|20-20|,|20-1|\}=19$

\section{Figure 2. An Example of the Homogeneity Operator}

where $I(x, y)$ is center pixel and $I$ is new image showing edges. This operator gives a good rendition of the edges in the original image. 


\subsection{Spatial Region Periodicity}

This paper used spatial region periodicity HOG descriptors based on dense and overlapping encoding of small spatial image regions (cells) and large spatial image regions (blocks). First, each cell contains orientation bins. In general, cell characteristic values obtained from each cell are expressed by concatenation method, as multi dimension cell space, which are equal to Equation (7) and Figure 3 (a):

$$
\Gamma=\left[\begin{array}{llll}
C_{1} & C_{2} & \ldots & C_{n}
\end{array}\right]
$$

This paper adapted cell histogram features saved at an interval of cell number as shown in Figure 3 (b), unlike the general method. For example, if each cell has $8 \times 8$ pixels for original image (128x64), the number of cell is 128 . And the features are saved by 128 periods. The method used in this study to save feature vectors is to save feature vectors using the thinned out method for each cell, according to cell numbers, as shown Figure 3 (b).

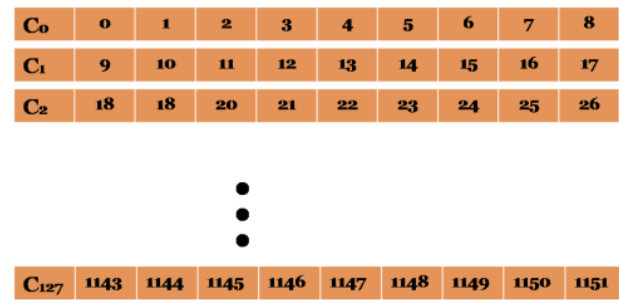

(a)

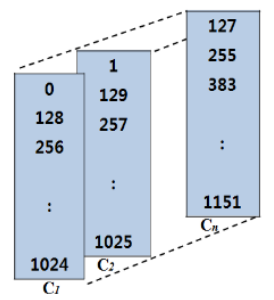

(b)

Figure 3. Cell Feature Vectors (a) Concatenation Method (b) Spatial Region Periodicity

Second, the block grid ( $\mathrm{n} \times \mathrm{n}$ ) which define the number of cells in each block is large spatial regions having overlapping. Generally block features are saved by concatenation method, as shown in Figure 4 (a). Before the block normalization, each block transposes to scatter the features according to the rule, as shown Figure 4 (b). Similar to the cells, we calculate the number of blocks. Then proposed block features are saved by the block period, as shown Figure 4 (c).

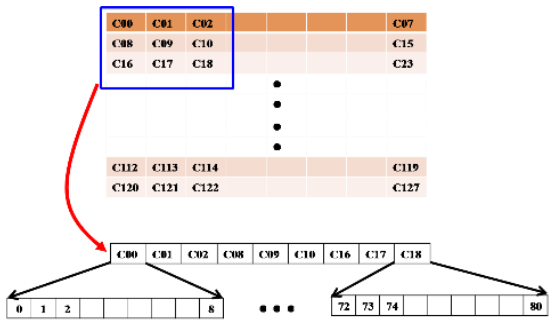

(a)
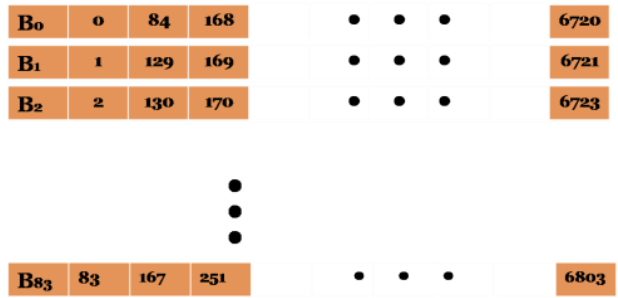

(c)

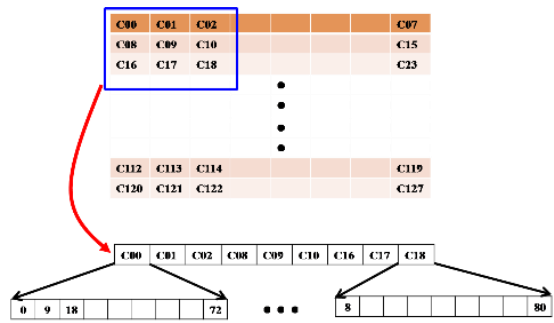

(b)

Figure 4. Block Feature Vectors (a) the Location of General Block Features (b) Transpose of Block Features (c) Proposed Block Features using Block Periodicity 
Characteristic vectors are saved in the position information within each of the cells and blocks. The images used in this study are expressed by utilizing the characteristic vector saving positions and the matrix positions that each of the cells has, of which are shown in Figure 4 (c). Therefore, each of the cells and blocks has a scattering characteristic vector at positions with same interval for dense and overlapping processing, which is placed through whole cells and blocks.

\section{Classifier}

Adaboost is short for "adapted boosting", it is formulated by Yoav Freund and Robert Schapire [17]. Adaboost is an algorithm which makes a strong classifier from weak classifiers. It is a simple learning algorithm that selects a small set of weak classifiers from a large number of potential features according to the weighted majority of classifiers. The training procedure of Adaboost is a greedy algorithm, which constructs an additive combination of weak classifiers. Our boosting algorithm is basically the same as P. Viola's algorithm [18]. The algorithm takes as input a training set where each belong to some domain and each label is in some label set. The algorithm takes as input a training set $\left(x_{1}, y_{1}\right), \cdots,\left(x_{n}, y_{n}\right)$ where each $x_{i}$ belong to some domain $X$ and each label $y_{i}$ is in some label set $Y$.

Given training set: $\left(x_{1}, y_{1}\right), \cdots,\left(x_{n}, y_{n}\right)$

where $x_{i} \in X, \quad y_{i} \in Y=\{+1,-1\}$

1) Initialize weights $w_{1, i}=\frac{1}{2 m}, \frac{1}{2 l}$ for $y_{i}=+1,-1$

$m$ : the number of positive image (two-wheeler, +1 )

$n:$ the number of negative image (non two-wheeler, -1)

2) For $t=1 \cdots T$ :

(a) Normalize the weights,

$$
w_{t, i}=\frac{w_{t, i}}{\sum_{j=1}^{n} w_{t, j}}
$$

so that $w_{t, i}$ is a probability distribution of $i$ th training image for $t$ th weak classification

(b) For each feature, $\mathrm{j}$, train a classifier $h_{j}$ which is restricted to using a single feature.

The error is evaluated with respect to $w_{i}$

$$
\varepsilon_{j}=\sum_{i} w_{i}\left|h_{j}\left(x_{i}\right)-y_{i}\right|
$$

(c) Choose the classifier, ${ }^{h_{t}}$, with the lowest error $\varepsilon_{t}$

(d) Update the weights: $w_{t+1, i}=w_{t, i} \beta_{t}^{1-\varepsilon_{i}}$ where $\varepsilon_{i}=-1$ if example ${ }^{x_{i}}$ is classified correctly, ${ }^{\varepsilon_{i}=+1}$ otherwise,

and

$$
\beta_{t}^{i}=\frac{\varepsilon_{t}}{1-\varepsilon_{t}}
$$

3) Output the final hypothesis:

$$
H(x)=\operatorname{sign}\left(\sum_{t=1}^{T} \alpha_{t} h_{t}(x)\right) \text {, where } \alpha_{t}=\log \left(1 / \beta_{t}\right)
$$


In stage 1, initial input value $\mathrm{x}$ represents the training feature for image and $\mathrm{y}$ represents classification of positive $(+1)$ and negative $(-1)$, like as positive and negative image.

In stage 2 , it conducts initial and updates the weighting factors.

In stage 3, it creates weak classifiers, in which it selects a weak classifier having a minimum error and imposes a weighting factor to the classifier, when weighting factor $w(j)$ will be increased for an incorrectly classified training image but weighting factor $w(j)$ will be decreased for a correctly classified training image, in order for initially selected characteristics to take a role to classify easily, but later selected characteristics will take the role of more difficult classification.

The final hypothesis $H$ is a weighted majority vote of the $\boldsymbol{T}$ weak hypotheses where $\alpha_{t}$ is the weight assigned to $h_{t}$.

Using two strong classifications, this paper suggests the $2^{\text {nd }}$ stage cascade method. It improves the recognition rate due to the complementary role for the two feature vector of quite different types.

\section{Experimental Results}

To access the effectiveness of the proposed evolutionary method, the algorithm was applied to a practical proposed method. In this study, an experiment was carried out with an ordinary user computer environment consisting of a Pentium 5.1 GHz (6GB DDR Memory) and Visual C++ 6.0 program. An image of two-wheelers can be expressed with various angles in an automobile. For our purposes, it is hypothesized in the experiment in 2 cases, i.e., a two-wheeler is running in front of an automobile (rear appearance) and a two-wheeler is coming toward the automobile (front appearance). And the experiment was done for the attitude of 90 degrees and the attitude of within 60 degrees in basis of horizontal line. 2,353 pictures of normalized two-wheelers were used with a size of $64 \times 128$ (width $\mathrm{x}$ height) from the taken photos with the size of $640 \times 480$ and collected from the internet, as shown in Table 1 . They were utilized by dividing a training image and an experimental image. Non two-wheelers were obtained by utilizing randomly extracted pictures from the streets of ordinary cities and uptown. The number of non-twowheelers used in the training was equal to the number of two-wheelers, and 3,000 pictures of non-two-wheelers were used in the experiment.

Table 1. Number of Training and Test Images

\begin{tabular}{|c|c|c|c|c|c|}
\hline \multicolumn{2}{|c|}{ Angle } & Kind & Front & Rear & Total \\
\hline \multirow{4}{*}{$60^{\circ}$} & \multirow{2}{*}{ Bicycle } & Training & 160 & 145 & 305 \\
\hline & & Test & 190 & 150 & 340 \\
\hline & \multirow{2}{*}{$\begin{array}{l}\text { Motor- } \\
\text { cycle }\end{array}$} & Training & 40 & 40 & 80 \\
\hline & & Test & 46 & 50 & 96 \\
\hline \multirow{4}{*}{$90^{\circ}$} & \multirow{2}{*}{ Bicycle } & Training & 428 & 435 & 863 \\
\hline & & Test & 430 & 415 & 845 \\
\hline & \multirow{2}{*}{$\begin{array}{l}\text { Motor- } \\
\text { cycle }\end{array}$} & Training & 102 & 117 & 219 \\
\hline & & Test & 119 & 115 & 234 \\
\hline
\end{tabular}




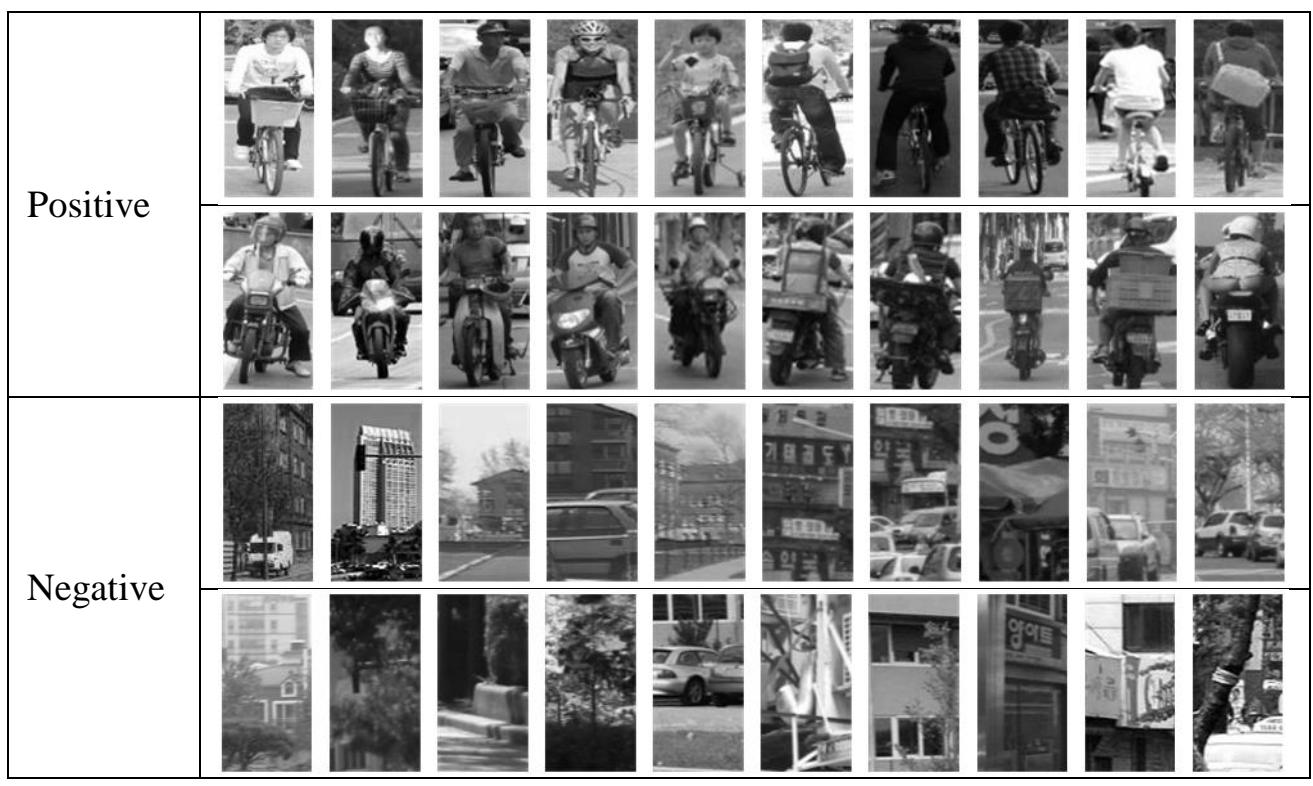

Figure 5. Example of Positive and Negative Images

An example of images used in experiment is shown in Figure 5. In Figure 5, the first row refers to the images of two-wheelers corresponding to bicycles and the second row refers to the images corresponding to the motorcycles. And the third and fourth row refers to negative images which were collected randomly in the images.

The experiment was carried out using HOG Method (the most widely utilized), homogeneity method, and modified HOG (G-HOG), which is calculated by the gamma correction. A range of thresholds of -20 to 20 was utilized in classification, and confusion matrix, true positive rate (TPR) and false positive rate (FPR) were used for analyzing experimental results per angles for the methods, and ROC [14] curves are shown in Figure 6, by applying Equation (8) below:

$$
T P R=\frac{T P}{T P+F N} \quad F P R=\frac{F P}{F P+T N}
$$

where "TP" is True Positive", "FP" is False Positive", "TN" is True Negative and "FN" is False Negative.

In Figure 6, "Moto" means motorcycle, "Bike" means bicycle, and "MB" is a mixture of motorcycles and bicycles, respectively. Also, the numerals behind each of the abbreviations " 60 " signifies within 60 degrees, "90" within 90 degrees, and "90-60" a mixture of 90 and 60 degrees, respectively, as well.

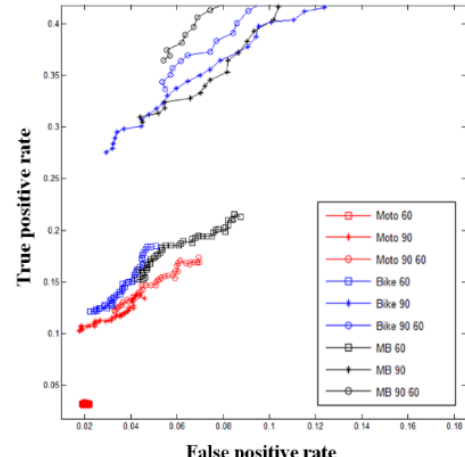

(a)

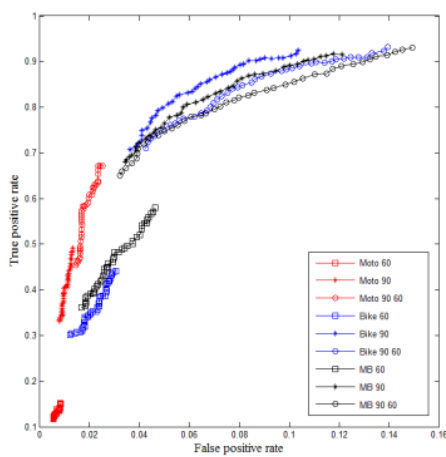

(b) 


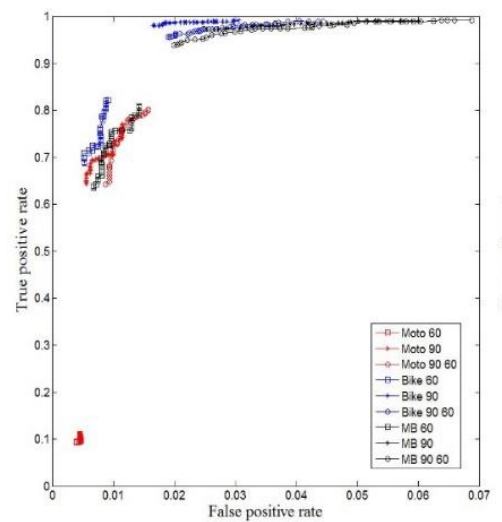

(c)

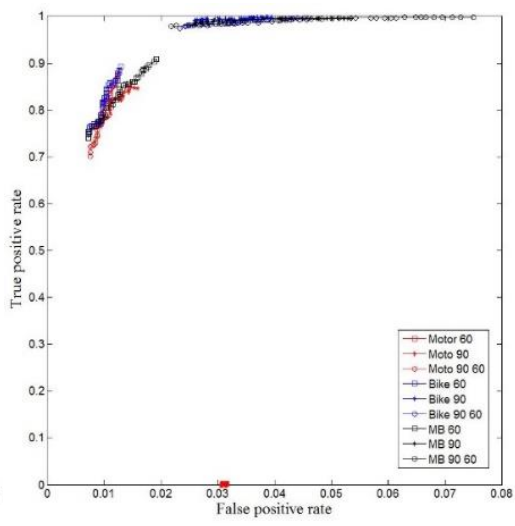

(d)

\section{Figure 6. Experiment Results (a) Results of Ordinary HOG Method (b) The result of Local Binary Pattern (LBP) (c) Results of the Homogeneity Method (d) Results of the G_HOG Method by Applying the Suggested Algorithm}

The motivation of the proposed method is the Sobel operator that the center of kernel has twice value than others. So for the proposed method, we applied new magnitude calculation method adapted gamma correction algorithm by applying Equation (5)

$$
\begin{gathered}
M^{\prime}=M \times(1 / \gamma) \quad H^{\prime}=H \times(\gamma) \\
\text { where } \\
\gamma=\left\{\begin{array}{c}
0.5 \text { if } \quad M>\text { th } \\
5 \quad \text { else }
\end{array}\right.
\end{gathered}
$$

Finally the feature calculation is

$$
F=\left\{\begin{array}{l}
M^{\prime} \quad \text { if } \quad M^{\prime}>a v g_{c} \\
H^{\prime} \text { else }
\end{array}\right.
$$

where $a v g_{c}$ is the average of histogram bin in the block.

In Figure 6 (a), it has shown that the experiment according to "MB" has the best results among these experiments, but the recognition rate is significantly low. However, Figure 6 (c) shows that the results of the MB experiments according to the general homogeneity method have a higher recognition rate than adapted concatenation HOG method, using Equation (6). When applied with another analytical method, spatial region periodicity, as shown in Figure 6 (c) and (d), because the area of the curve of the suggested method is larger than that of the curve of the concatenation HOG method, it is known that this system has a better performance. When G_HOG algorithm was applied for the other characteristic vector (homogeneity) method, a higher recognition rate could be obtained, and the results are listed in Figure 6 (d). The highest accuracy for each of the methods was calculated with equation (10) and the results are listed in Table 2. For the same database, a comparison of the performance results for other different feature types (LBP) is shown in Figure 6 (b). The result of LBP features shows lower performance than HOG and the proposed algorithm, because of a concentrated lower area in the Figure 6 (b) and Table 2.

$$
\text { Accuracy }=\frac{T P+T N}{T P+F P+T N+F N}
$$

As shown in Table 2, Bike has a higher accuracy than Moto (motorcycle) and MB for the angle 60 and 90, signifying that bikes have a trend of better classifying characteristics than motorcycles. In our opinion, a bike is more simple structure than a motorcycle. And it has no loading of baggage at the rear or by the high loading of baggage. In the experiment of the mixture of the two kinds of two-wheelers, MB, 90 and 60 , the result of the suggested method accuracies also has a higher accuracy than the existing algorithm. 
Because two-wheelers are composed of persons, bicycles, or motorcycles together with various shapes of baggage, the upper part of a waist is similar to a pedestrian but the lower part is diversified and complicated in shape, so that it becomes another field of challenge besides detection of pedestrians and automobiles for an intelligent automobile. The two-wheeler in the following means a combination of a person and a machine.

Table 2. Accuracies for Each of the Methods (\%)

\begin{tabular}{|c|c|c|c|c|c|}
\hline \multicolumn{2}{|c|}{ Method } & HOG & LBP & H_HOG & G_HOG \\
\hline \multirow{3}{*}{$\mathbf{6 0}$} & $\boldsymbol{M}$ & 61.1 & 53.7 & 81.66 & 81.75 \\
\cline { 2 - 6 } & $\boldsymbol{B}$ & 71.2 & 72.2 & 97.37 & 97.85 \\
\cline { 2 - 6 } & $\boldsymbol{M} \boldsymbol{B}$ & 76.7 & 71.1 & 96.40 & 97.31 \\
\hline \multirow{3}{*}{$\mathbf{9 0}$} & $\boldsymbol{M}$ & 74.9 & 48.0 & 97.24 & 97.70 \\
\cline { 2 - 6 } & $\boldsymbol{B}$ & 78.3 & 77.8 & 98.32 & 97.75 \\
\cline { 2 - 6 } & $\boldsymbol{M} \boldsymbol{B}$ & 76.1 & 74.5 & 97.43 & 97.67 \\
\hline \multirow{3}{*}{$\mathbf{9 0 - 6 0}$} & $\boldsymbol{M}$ & 77.8 & 71.8 & 96.70 & 97.76 \\
\cline { 2 - 6 } & $\boldsymbol{B}$ & 75.5 & 73.4 & 97.53 & 97.65 \\
\cline { 2 - 6 } & $\boldsymbol{M} \boldsymbol{B}$ & 73.1 & 72.0 & 97.00 & 97.83 \\
\hline
\end{tabular}

\section{Conclusion}

Detecting accurate and efficient two-wheelers from still images is one of the most difficult works for intelligent vehicles due to the variety of poses, environmental conditions, and cluttered background images. Classification based methods have comprised the mainstream of research and have been shown to achieve successful results in object detection via two-wheeler detection. In this study, we have introduced a novel practical implementation of the solution for weak object (vulnerable road users) on the road using homogeneity operator and modified histogram of oriented gradients using spatial region periodicity and the Adaboost classifier. The underlying motivation of our approach originated from the observation that homogeneity operator and gamma correction have the simplest and quickest edge detection, contrary properties, and are robust against variation in pose or illumination.

It has been experimentally demonstrated that the proposed method as new magnitude value, spatial region periodicity, and cell average by the gamma correction leads to better classification results than other traditional methods from ROC. The Adaboost classification has comprised the main stream of research for the detection of two-wheelers and the results compared with the existing method have shown that it is a highly improved system performance. Additionally, among the several proposed methods, the G-HOG method showed a higher detection rate for mixed angle (90-60) than others.

For the further research, we will consider including occluded regions, changed objects according to the weather and night environment.

\section{Acknowledgments}

This paper is a revised and expanded version of a paper entitled "Homogeneity Operator and Modified Histogram of Oriented Gradients based Two-Wheeler Detection Algorithm Development using Adaboost" presented at MITA2017, Kuala lumpur, July 911, 2017. And this work was supported by a grant from 2017 Research Funds of Korea Western Power Co., Ltd. (No. 2017-0142). 


\section{References}

[1] Y. Lee, T. Kim and J. Shim, "Two-wheeler Detection System using Histogram of Oriented Gradients based on Local Correlation Coefficients and Curvature", Journal of Multimedia Information System, vol. 2, no. 4, (2015), pp. 303-310.

[2] H. Jung, Y. Ehara, J. K. Tan, H. Kim and S. Ishikawa, "Applying MSC-HOG Feature to the Detection of Human on a Bicycle", 12th International Conference on Control, International Conference on Control, Jeju Island, South Korea, (2012).

[3] T. Gandhi and M. M. Trivedi, "Pedestrian Protection Systems: Issues, Survey, and Challenges", IEEE Transaction on Intelligent Transportation Systems, vol. 8, no.3, (2007), pp.413-430.

[4] L. Yu, F. Zhao and Z. an, "Locally Assembled Binary Feature with Feed-forward Cascade for Pedestrian Detection in Intelligent Vehicle", International conference on Cognitive Informatics, Beijing, China, (2010).

[5] Q. Zhu, M. C. Yeh, K. T. Cheng and S. Avidan, "Fast human detection using a cascade of histogram of oriented gradients", IEEE Conference on Computer Vision and Pattern Recognition, New York, USA, (2006).

[6] T. Watanabe, S Ito and K. Yokoi, "Co-occurrence histogram of Oriented Gradients for Detection", Advances in Image and Video Technology, vol.5414, (2009), pp. 37-47.

[7] X. Y. Wang, T. X. Han and S. Yan, "An HOG-LBP human detector with partial occlusion handling", International conference on computer Vision, Kyoto, Japan, (2009).

[8] Y. Lee, S. Lee, T. Kim and J. Shim, "New approach to twowheelers detection using Cell Comparison", Journal of Multimedia Information System, vol.1, no.1, (2014), pp. 45-54.

[9] O. Tuzel, F. Porikli and P. Meer, "Human detection via classification on Riemannian manifolds", Computer Vision and Pattern Recognition, Minneapolis, USA, (2007).

[10] P. F. Felzenzwalb, R. B. Girshick, D. Mcallester and D.Ramanan, "Object Detection with Discriminatively Trained Part-based Models", IEEE Transaction on Pattern Analysis and Machine Intelligence vol.32, no.9, (2010), pp.1627-1645.

[11] G. zhenhua, Z. Lei and D. Zhang, "A completed Modeling of Local Binary pattern Operator for Texture Classification”, IEEE Transaction on Image Processing, vol 19, no, 6, (2010), pp.1657-1663.

[12] T. Ahonen, A Hadid and M. Pietikainen, "Face Description with local binary patterns: Application to face recognition", IEEE Transaction on Pattern Analysis and Machine Intelligence, vol28, no.12, (2006), pp2037-2041.

[13] K. Song, Y. Yan, W. Chen and X. Zhang, "Research and Perspective on Local Binary Pattern", Acta Automation sinica, vol39, no.6, (2013), pp.730-744.

[14] H. Cho, P. E. Rybski and W. Zhang, "Vision-based bicyclist detection and tracking for intelligent vehicles", IEEE Intelligent Vehicle Symposium, San Diego, USA, (2010).

[15] N. Dalal and B. Triggs, "Histogram of Oriented Gradients for Human Detection", 2005 Computer Vision and Pattern Recognition, San Diego, USA, (2005).

[16] D. Phillips, "Image Processing in C: Analyzing and Enhancing Digital Images", R\&D Publication, Rohtak, (1994).

[17] http://en/wikipedia.org/wiki/Adaboost, (2017).

[18] P. Viloa, M. Jones and M. Snow, "Detecting pedestrians using patterns of motion and appearance", 9th International Conference on Computer Vision, Nice France, (2003).

[19] http://en/wikipedia.org/wiki/Receiver_operating_characteristic, (2017).

\section{Authors}

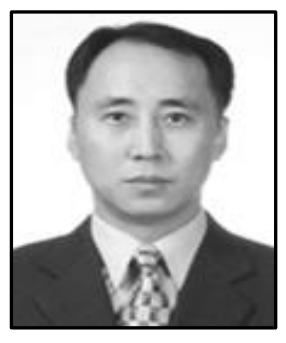

Yeunghak Lee, he received his Ph.D degree from Yeungnam University, Korea, in 2003. He had one year experience at University of Cardiff as postdoc research fellow. He was a professor of department of avionic electronic engineering at Kyungwoon University. He is currently working as a senior researcher in the Department of Computer Science at Andong National University. He contributes as a management editor for the Journal of Multimedia and Information Systems. His research interests include pattern recognition, embedded system and computer vision. 


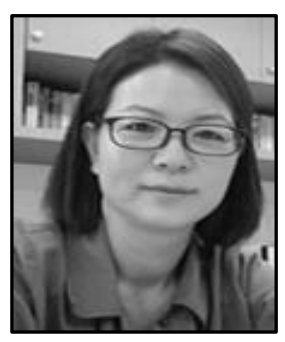

Yunju Jung, she received the B. S. degree in 1991 from Andong National University. Currently she studies for Ph.D. at Department of Computer Engineering, Kyungbook National University, Korea. Since 1998, she works as a lecturer in Andong National University. Her research interests include computer vision, image processing, and pattern recognition, Deep Learning, IoT and Networking.

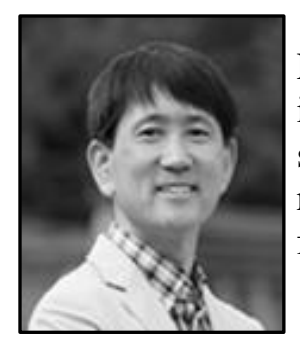

Jaechang Shim, he is a Professor in the Department of Computer Engineering at Andong National University. His research interests include pattern recognition, image processing, embedded vision system, biometric authentication, and 3D object recognition. $\mathrm{He}$ received the best paper awards from IAPR in 1998. He has been a cofounder of PAMI Inc. since 1998. 